"Asymmetric information, strategic transfers, and the design of long-term care policies"

Chiara Canta and Helmuth Cremer 


\title{
Asymmetric information, strategic transfers, and the design of long-term care policies*
}

\author{
Chiara Canta \\ Department of Economics and Finance \\ TBS Business School \\ 31068 Toulouse, France \\ Helmuth Cremer \\ Toulouse School of Economics, \\ University of Toulouse Capitole \\ 31015 Toulouse, France
}

October 2020

*Financial support from the Chaire "Marché des risques et creation de valeur" of the FdR/SCOR is gratefully acknowledged. Helmuth Cremer gratefully acknowledges the funding received by TSE from ANR under grant ANR-17-EURE-0010 (Investissements d'Avenir program). 


\begin{abstract}
We study the design of social long-term care (LTC) insurance when informal care is exchange-based. Parents do not observe their children's cost of providing care, which is continuously distributed over some interval. They choose a rule specifying transfers that are conditional on the level of informal care. Social LTC insurance is designed to maximize a weighted sum of parents' and children's utility. The optimal uniform public LTC insurance can fully cover the risk of dependence but parents continue to bear the risk of having children with a high cost of providing care. A nonlinear policy conditioning LTC benefits on transfers provides full insurance even for this risk. Informal care increases with the children's welfare weight.

Our theoretical analysis is completed by numerical solutions based on a calibrated example. In the uniform case, public care should represent up to $40 \%$ of total care but its share decreases to about $30 \%$ as the weight of children increases. In the nonlinear case, public care increases with the children's cost of providing care at a faster rate when children's weight in social welfare is higher. It represents $100 \%$ of total care for the families with high-cost children.
\end{abstract}

JEL classification: $\mathrm{H} 2, \mathrm{H} 5$.

Keywords: Long-term care, informal care, strategic bequests, asymmetric information. 


\section{Introduction}

Currently, informal care provided by family members represents a significant part of long-term-care services. Since it is by definition informal its exact level is hard to quantify but it is believed to be immense; see Bonsang and Schoenmaeckers (2015) and Norton (2016, Section 3) for an overview of the relevant empirical studies. For instance, Bolin et al. (2008) use SHARE (Survey of Health, Aging, and Retirement in Europe) data and find that elderly individuals with at least one child are likely to receive some informal care.

Informal care has a number of attractive features. The family provides services that are "warm", and appear to be inexpensive. However, it also has deficiencies and limitations, as its true costs, in particular, are often hidden. Caregiving can be extremely costly for the caregivers, and the costs may be underestimated by parents. As Norton (2016, page 961) puts it, "The economic cost of informal caregiving would be even larger if the cost included the deleterious effects on caregivers' health and labor market outcomes." This aspect is well documented in the medical literature. ${ }^{1}$ In the economic literature, however, it has been neglected: exceptions are Colombo et al. (2011) or Coe and Van Houtven (2009). For parents, the major shortcoming of informal care is that its availability is subject to some randomness and asymmetric information. Fertility cannot be fully controlled and the children's type, that is, their cost of providing care (or their degree of altruism), may not be known to parents at the time they make their LTC arrangements.

Since private insurance markets for LTC are very thin, the shortcomings of informal care create a potential role for a more coordinated and better designed public intervention through social LTC provision or insurance. However, LTC policies are likely to affect family transfers, and informal care, in particular. Consequently, policy design must take into account its effects on exchanges within the family. These in turn depend

\footnotetext{
${ }^{1}$ For a survey, see Brodaty and Donking (2009). They point out that "The effects of being a family caregiver, though sometimes positive, are generally negative, with high rates of burden and psychological morbidity as well as social isolation, physical ill-health, and financial hardship," and that "The high levels of burden and psychological morbidity are well documented, as are factors that predict which caregivers are vulnerable to these."
} 
on the motivations of informal caregivers.

In a fairy tale world, informal care would be motivated by altruism. However, in reality, other factors appear to be at work. Care may be "bought" through implicit exchanges or be "imposed" by social norms. These three motives have been shown to coexist and their relative importance to depend on the social and family context. ${ }^{2}$ In this paper, we focus on exchange-based transfers. Informal care is compensated for by monetary transfers, gifts or bequests. ${ }^{3}$ While the model does not explicitly take altruism into account, altruism can have an impact on the children's cost of providing care. Specifically, we assume that children differ in their preferences, which may depend on the degree of altruism, the strength of family bonds and many other factors, such as the children's exact working schedule, income and other commitments. These in turn will affect their cost of providing care.

Canta et al. (2016) and Canta and Cremer (2020) also assume that children are heterogeneous and that their type is unknown to parents ex ante. However, these papers rule out transfers from parents to children, and informal care is exclusively motivated by altruism. Parents cannot "buy" extra care through gifts or bequests. The current paper, on the other hand, concentrates on families (or societies) where children's altruism is not sufficient, so that parents still need to "buy" extra care through gifts or bequests. The relevance of the exchange-based provision of informal care has been widely documented empirically. Cox and Rank (1992) using US data, show a positive correlation between transfers from parents to children and both informal care and children's income, which supports the exchange motive. Light and McGarry (2004) find both altruistic and exchange-motivated transfers in a survey of US mothers who have left unequal bequests. In a Danish interview study, Lewinter (2003) documents the importance of reciprocity (small gifts and hospitality) for both caregivers and dependent parents. Ciani and Deiana (2018) also provide evidence of reciprocity in Italian data. Alessie et al. (2014) and Tomini et al. (2016) use SHARE data and show that exchange motives are important alongside altruism, and that the strength of altruism or exchange

\footnotetext{
${ }^{2}$ For a detailed survey of the empirical literature, see Arrondel and Masson (2006).

${ }^{3}$ See for instance Kotlikoff and Spivak (1981) and Bernheim et al. (1985).
} 
motives depends on family and individual characteristics. More generally, studies based on SHARE data (see for instance Klimaviciute et al., 2017) find a lot of variation in terms of informal care motivations across Europe.

This paper is inspired by Canta and Cremer (2019), which we extend and generalize by considering a continuous distribution of the children's heterogeneity parameter rather than a two-type model. From a theoretical perspective, this enables us to provide a much richer analysis of both the parent's and the government's optimal policy. Specifically, we can characterize the full profile of the induced informal care as a function of the child's cost of care. Even more importantly, the continuum model is better suited to being tested empirically. We illustrate this by providing calibrated numerical examples that show what proportion of total care should be provided publicly and how significant its impact on social welfare is. The example also allows us to fully characterize the nonlinear policy that implements the optimal allocation by relating public care to the parents' transfer.

We assume that parents can commit to a transfer, gift or bequest, conditional on the level of informal care provided. While our results do not depend on whether the transfer is a gift or a bequest, the use of gifts as payments is more relevant empirically. Children who provide informal care have been shown to receive larger inter vivos transfers (Koh and MacDonald, 2006; Norton and Van Houtven, 2006; Norton et al., 2013). In contrast, bequests are usually divided equally among children (Menchik, 1980; Tomes, 1981). In Europe, most countries impose equal sharing rules by law. Even in the US, where, in principle, estates can be unequally shared, bequests tend to be divided equally even if children provide unequal amounts of informal care (Norton and Taylor, 2005). ${ }^{4}$

As in Canta and Cremer (2019), parents do not perfectly know their children's preferences, and their cost of providing informal care. Consequently, children may try to overemphasize their costs in order to obtain a larger transfer and to extract a rent. In this case, parents cannot absorb the full surplus generated by the intrafamily exchange. Even when they can use nonlinear rules to screen for the children's cost parameter, they

\footnotetext{
${ }^{4}$ Inter vivos transfers are also more appealing theoretically as payment of informal care. Gifts "can be adjusted quickly to the amount of care, are less costly than writing a will, and can be kept secret from other family members and the public." (Norton and Van Houtven, 2006)
} 
must leave a positive rent to all of their children whose cost of providing care is lower than the highest level. The care provided by children with costs above the minimum level is therefore distorted downwards in order to mitigate the rent enjoyed by those with lower costs.

Social LTC is designed to maximize a weighted sum of parents' and children's utilities. It thus explicitly takes into account the caregivers' wellbeing, in contrast to the existing literature, which has mostly concentrated on parents' welfare. Since our focus is on LTC policies and not on the more general issue of intergenerational redistribution, we assume throughout the paper that the government cannot redistribute between parents and children using lump-sum taxes. ${ }^{5}$

We first characterize the optimal uniform social LTC policy, providing a given LTC transfer to all dependent individuals. The transfer is financed by a uniform lump-sum tax levied on parents. We show that this policy affects the levels of informal care as well as the transfers to children. In other words, the distortions of informal care, and thus the distribution of rents between parents and children, depend on the level of the public LTC transfer. If the social planner only cares about parents, the risk of dependence is fully insured. As the weight of children in the social welfare function (SWF) increases, the insurance coverage of parents is adjusted in order to enhance the rent enjoyed by the caregivers. The risk of having a high-cost child is never completely insured.

We then turn to the study of general policies. We assume that transfers from parents to children are publicly observable and that LTC benefits can be conditioned on these transfers. ${ }^{6}$ This general LTC policy can then screen for the children's cost parameter, even if the level of informal care is observable only to parents. Technically, we have a "nested" principal-agent problem where the policy can screen for the children's cost of providing care, but only indirectly via the parents. ${ }^{7}$ Even if parents do not observe their children's cost of providing care, they have superior information since they observe

\footnotetext{
${ }^{5}$ Since we have a model with quasi-linear preferences, allowing for such taxes would yield corner solutions, where the entire surplus would be given to the generation with the highest welfare weight.

${ }^{6}$ In practice, inter vivos gifts and bequests (estates) are already taxed albeit for different reasons (Cremer and Pestieau, 2006).

${ }^{7}$ See Guesnerie and Laffont (1978) in the context of regulation and Tirole (1986) in the context of hierarchies.
} 
informal care. We show that the general policy can provide full insurance to parents, even against the risk of having high-cost children.

One surprising implication of our model is that the optimal level of informal care increases as the children's weight in the social welfare function increases. The explanation for this result is that children receive a compensation that increases with the level of informal care they provide. The policy, in order to increase the children's rents, tends to mitigate the downward distortion introduced by their parents.

Finally, we provide numerical solutions based on a calibrated example. We show that in the uniform case and when market care is not available, public care should represent about $40 \%$ of total care when the SWF includes only the parents' utility. The share of public care decreases to about $30 \%$ as the weight of children increases. In the nonlinear case, public care increases with the children's cost of providing care at a rate that is larger, the larger the weight of children in social welfare. It represents the full amount of total care for families with high-cost children when the weight of children in the SWF is zero. These results are mirrored by the properties of the level of social care as a function of the transfer that implements the optimal policy. Not surprisingly it is a decreasing function, because parents with lower-cost children buy more informal care in exchange for larger transfers (and thus "need"less social care). In our example, we also find that this function decreases at a lower rate as the weight of children in the SWF increases. Intuitively, if the social planner cares a lot about children's wellbeing, social care should not decrease too fast as intrafamily transfers increase, in order to ensure that parents elicit (and pay) a large amount of informal care.

The paper is structured as follows. In Section 2, we describe the model. In Sections 3 and 4 we characterize the optimal uniform and nonlinear policy, respectively, while section 5 presents the results of our calibrated example.

\section{Model}

We consider a generation of parents who are ex ante identical. When they are young, they all receive an exogenous income $w \bar{T}$ and allocate it between consumption $c$ and savings $s$. When old, they are dependent with probability $\pi$ and healthy with probability 
$(1-\pi)$. We assume that their utility function is quasi-linear in young age consumption, so that their expected utility can be written as

$$
E U=w \bar{T}-s+(1-\pi) U(s)+\pi E[H(m)]
$$

where $E$ is the expectation operator, and $m=s+a-\tau(a)$ is consumption when old and dependent. In case of severe dependence, one can think of $m$ as consumption of LTC services. In the case where parents are dependent, in addition to their savings, they receive care from their children, $a$, in exchange for a transfer $\tau(a)$. In milder cases $m$ includes LTC, where both the care bought on the market and informal care are expressed in monetary terms. The healthy elderly simply consume their savings. We assume that $U^{\prime}>0, H^{\prime}>0, U^{\prime \prime}<0, H^{\prime \prime}<0$, and that both functions satisfy the Inada conditions. Furthermore, we assume that, for all $x, U^{\prime}(x)<H^{\prime}(x)$. This ensures that there is a role for insurance against the risk of dependence.

In line with the literature on strategic bequests, we allow the transfer rule to depend on the level of informal care received from children, and we assume that parents can commit to such a rule. However, in contrast to the traditional strategic transfer literature, we assume that parents do not have full information on their children. Specifically, they cannot observe the children's cost of providing informal care, which is represented by a parameter $\beta$. This parameter is determined by different factors affecting the provision of informal care, such as the children's degree of altruism or the children's opportunity cost of providing care. We assume that $\beta$ is distributed over the interval $[\beta, \bar{\beta}]$ according to the c.d.f. $F(\beta)$.

When parents are healthy, children do not provide any care, and their utility is normalized to zero. When parents are dependent, children's utility is equal to

$$
U_{k}=c_{k}-v(a, \beta) \geq 0
$$

where children's consumption $c_{k}=\tau(a)$, that is the transfer from their parents, while $v(a, \beta)$ represents the cost of providing care, with $v_{a}>0, v_{\beta}<0, v_{a a}>0, v_{a \beta}<0$. In words, the cost of providing care is increasing and convex in the level of care. It decreases in $\beta$, so that children displaying higher $\beta$ s are the "good types" for whom 
providing care is less costly. Furthermore, the marginal cost of providing informal care decreases when $\beta$ increases.

The level of care chosen by a child of type $\beta, a(\beta)$, is obtained by maximizing (1), and is implicitly defined by

$$
\tau^{\prime}(a(\beta))=v_{a}(a(\beta), \beta) .
$$

The level of care depends on both the cost parameter and the bequest function $\tau($.).

Each parent has one child. Parents anticipate their children's behavior, but do not observe their cost parameter $\beta$. Their problem is then to maximize with respect to $s$ and $\tau(a)$ the following expected utility

$$
E U=w \bar{T}-s+(1-\pi) U(s)+\pi E_{\beta}[H(s+a(\beta)-\tau(a(\beta))]
$$

We can rewrite this problem as a problem where parents choose $s, a(\beta)$, and $\tau(\beta)$ to maximize

$$
E U=w \bar{T}-s+(1-\pi) U(s)+\pi E_{\beta}[H(s+a(\beta)-\tau(\beta)],
$$

subject to the children's participation and incentive constraints.

We will study the optimal public LTC insurance policy. The policy will consist of a transfer to dependent parents financed through a lump-sum tax paid by parents when young. The timing is as follows. The government commits to a policy in period 1 . In period 2, parents observe the LTC policy and commit to a transfer rule $\tau(a)$. Finally, in period 3, children choose the level of informal care $a$ according to (2).

When we consider nonlinear policies, the LTC transfer can be conditioned on the bequest level $\tau$, but not on the level of informal care, which is not publicly observable. The optimal policy maximizes a weighted sum of parents' and children's welfare. The weight of children in the social welfare function is equal to $\alpha \in[0,1]$, and the weight of parents is equal to $(1-\alpha)$.

We will first consider a uniform public LTC transfer, and then relax this assumption and allow the public transfer to depend on bequests. In both cases, we first present the benchmark case where there is no asymmetry of information between children and parents, and characterize the corresponding LTC policy. We refer to this as "full infor- 
mation" for simplicity, but it must be emphasized that informal care is never publicly observable. We then turn to the asymmetric information case.

\section{Uniform LTC benefit}

We first consider the case where the government uses a uniform LTC transfer. This case can be relevant in practice since the government may be unable to observe bequests or inter vivos gifts. In addition, uniform transfers may be used in practice for political reasons since nonuniform policies may fail to gather sufficient political support; see for instance Casamatta et al. (2000) or Cremer and Pestieau (2018). In the following section, we will study a more sophisticated policy under which the LTC benefit can be conditioned on the level of transfers, which will allow the government to screen for different levels of $\beta$. In the remainder of this section, we first characterize the optimal uniform policy when parents observe their children's type. Whether or not the government also observes $\beta$ is irrelevant when we consider a uniform policy which, by definition, cannot be conditioned on $\beta$. We then turn to the case with asymmetric information between parents and children.

\subsection{Full information benchmark}

Assume first that the children's cost parameter is observable both by the parents and by the government. The problem of the parents is

$$
\begin{aligned}
\max _{s, \tau(\beta), a(\beta) \forall \beta \in[\underline{\beta}, \bar{\beta}]} & w \bar{T}-\pi g-s+(1-\pi) U(s) \\
& \left.+\pi \int_{\underline{\beta}}^{\bar{\beta}} H(s+a(\beta)-\tau(\beta)+g)\right) f(\beta) d \beta \\
\text { s.t. } & \tau(\beta)-v(a(\beta), \beta) \geq 0 \quad \forall \beta \in[\underline{\beta}, \bar{\beta}],
\end{aligned}
$$

where (3) represents the children's participation constraints. Under full information, these constraints are all binding. If one of them were not, the parents could increase their own utility by increasing $a(\beta)$ or decreasing $\tau(\beta)$. Substituting for $\tau(\beta)$ from (3), 
this problem can be rewritten as

$$
\begin{aligned}
\max _{s, a(\beta) \forall \beta} P^{f}= & w \bar{T}-\pi g-s+(1-\pi) U(s) \\
& \left.+\pi \int_{\underline{\beta}}^{\bar{\beta}} H(s+a(\beta)-v(a(\beta), \beta)+g)\right) f(\beta) d \beta .
\end{aligned}
$$

The first-order conditions of the parents are

$$
\begin{aligned}
& \frac{\partial P^{f}}{\partial a}=\pi\left[1-v_{a}(a, \beta)\right] H^{\prime}=0, \\
& \frac{\partial P^{f}}{\partial s}=-1+(1-\pi) U^{\prime}(s)+\pi \int_{\underline{\beta}}^{\bar{\beta}} H^{\prime} f(\beta) d \beta=0 .
\end{aligned}
$$

Using the first condition, we can write

$$
1=v_{a}(a, \beta) \quad \forall \beta \in[\underline{\beta}, \bar{\beta}] .
$$

Intuitively, with symmetric information, parents must reimburse their children exactly the cost of the informal care they provide. This ensures that the children's utility is just equal to their reservation level. Under full information there is no reason to pay more or, to use the terminology common in contract theory, to leave a rent to children. Parents will then choose the level of care that equalizes the marginal benefit and the marginal cost of informal care for each type $\beta$. In order to decentralize these levels of care, (2) shows that it is sufficient for parents to set $\tau^{\prime}(a)=1$. It is also possible to show that both $a$ and $m$ are increasing in $\beta$. Parents require higher levels of care and enjoy a higher consumption when dependent if they have lower-cost children.

Turning to the policy design, we now consider the optimal uniform LTC benefit $g$ financed with a lump-sum payment $\pi g$ paid by parents when young. When parents have full information, no rent will be left to children, so that the problem of the government can be simply written as

$$
\begin{aligned}
\max _{g} \quad G^{f}= & w \bar{T}-\pi g-s+(1-\pi) U(s) \\
& +\pi \int_{\underline{\beta}}^{\bar{\beta}} H(s+a-v(a, \beta)+g) f(\beta) d \beta,
\end{aligned}
$$


where $s$ and $a$ are the solutions to the parents' problem for any given $g$. Note that the children's welfare weight $\alpha$ plays no role in this expression because their utility is constant.

Using the envelope theorem, the first-order condition of the government is ${ }^{8}$

$$
\frac{\partial G^{f}}{\partial g}=-\pi+\pi \int_{\underline{\beta}}^{\bar{\beta}} H^{\prime} f(\beta) d \beta=0 .
$$

Combining this condition with (4) yields

$$
U^{\prime}(s)=\int_{\underline{\beta}}^{\bar{\beta}} H^{\prime} f(\beta) d \beta=1,
$$

implying that the marginal utility of consumption must be equalized across time and dependence states. However, the uniform transfer $g$ does not equalize consumption across parents with different types of children. In other words, $g$ cannot insure against the risk of having high-cost children. It is interesting to note that the levels of $a$ and $\tau$ under full information do not depend on the level of $g$.

It is also important to note that, in this benchmark case, public insurance would not be necessary if fair private LTC insurance were available. In that case, parents would all choose ex ante a level of private insurance equal to $g$.

\section{$3.2 \quad$ Asymmetric information}

In this section we depart from the standard strategic bequest model and assume that parents cannot observe the cost parameter of their children.

\subsubsection{Parents' problem}

The parents' problem is

$$
\begin{aligned}
\max _{s, \tau(\beta), a(\beta) \forall \beta \in[\underline{\beta}, \bar{\beta}]} \quad P^{a s}= & w \bar{T}-\pi g-s+(1-\pi) U(s) \\
& +\pi \int_{\underline{\beta}}^{\bar{\beta}} H(s+a(\beta)-\tau(\beta)+g) f(\beta) d \beta \\
& \\
\text { s.t. } \quad & \tau(\beta)-v(a(\beta), \beta) \geq \tau(\tilde{\beta})-v(a(\tilde{\beta}), \beta) \quad \forall \beta, \tilde{\beta} \in[\underline{\beta}, \bar{\beta}]
\end{aligned}
$$

$$
U_{k}(\beta)=\tau(\beta)-v(a(\beta), \beta) \geq 0 \quad \forall \beta,
$$

\footnotetext{
${ }^{8}$ We assume that the second order condition holds.
} 
where conditions (7) represent the incentive compatibility constraints, to ensure that individuals cannot benefit from reporting a type $\tilde{\beta}$ different from their true type $\beta$, while participation constraints are given by (8).

In Appendix A.1 we show that the set of incentive compatibility constraints in (7) can be replaced by the local incentive compatibility constraint $\dot{U}_{k}(\beta)=v_{\beta}(a(\beta), \beta)$, and by the monotonicity constraint $a^{\prime}(\beta) \geq 0$. Intuitively, children must be prevented from mimicking the type with the level of $\beta$ "just below" theirs and, together with the monotonicity condition, this ensures global incentive compatibility. A rent that is increasing in $\beta$ is then necessary to ensure that mimicking does not occur. With respect to the full information case, the utility of children is now positive and depends on their cost parameter. Furthermore, it may depend on the level of public insurance. In the Appendix A.1, we show that this utility can be expressed as

$$
U_{k}(\beta)=\int_{\underline{\beta}}^{\beta}-v_{\beta} d x .
$$

The parents' problem can then be rewritten as ${ }^{9}$

$$
\begin{aligned}
& \max _{s, a(\beta) \forall \beta \in[\underline{\beta}, \bar{\beta}]} \quad P^{a s}=w \bar{T}-\pi g-s+(1-\pi) U(s) \\
&+\pi \int_{\underline{\beta}}^{\bar{\beta}} H\left(s+a(\beta)-U_{k}(\beta)-v(a(\beta), \beta)+g\right) f(\beta) d \beta \\
& \text { s.t. } U_{k}(\beta)=-\int_{\underline{\beta}}^{\beta} v_{\beta} d x .
\end{aligned}
$$

We show in Appendix A.2 that the optimal levels of informal care are given by the following expression:

$$
1-v_{a}(a(\beta,) \beta)=\frac{-\int_{\beta}^{\bar{\beta}} H^{\prime} f(s) d s}{H^{\prime} f(\beta)} v_{a \beta}(a(\beta,) \beta)>0 \quad \forall \beta<\bar{\beta} .
$$

This condition implies that $a(\beta)$ is distorted downward with respect to its full-information level defined in (5) for all $\beta<\bar{\beta}$. This allows parents to reduce the rent enjoyed by low-cost children. Equation (11) also implies that for all $\beta<\bar{\beta}$, the optimal level of care $a(\beta)$ now depends on $g$ through the distortion term.

\footnotetext{
${ }^{9}$ In the following, we assume that that $a^{\prime}(\beta)>0$.
} 
We now characterize the optimal linear LTC policy. Recall that under asymmetric information between children and parents, children enjoy a rent, and their utility is positive. Consequently, they may enter the social welfare function, and the respective weights of parents and children will affect the optimal policy. The main trade-off in setting this policy will be between parents' insurance and children's utility.

\subsubsection{Optimal uniform policy}

The government's problem is given by

$$
\begin{aligned}
\max _{g} \quad G^{a s}= & (1-\alpha)\{w \bar{T}-\pi g-s+(1-\pi) U(s) \\
& \left.+\pi \int_{\underline{\beta}}^{\bar{\beta}} H\left(s+a(\beta)-v(a(\beta), \beta)-U_{k}(\beta)+g\right) f(\beta) d \beta\right\} \\
& +\alpha \pi \int_{\underline{\beta}}^{\bar{\beta}} U_{k}(\beta) f(\beta) d \beta,
\end{aligned}
$$

where $a(\beta), U_{k}(\beta)$, and $s$ are determined by the solution to the parents' problem.

Using the envelope theorem, the first-order condition of the government is ${ }^{10}$

$$
\frac{\partial G^{a s}}{\partial g}=(1-\alpha)\left\{-\pi+\pi \int_{\underline{\beta}}^{\bar{\beta}} H^{\prime} f(\beta) d \beta\right\}+\pi \alpha \frac{\partial \int_{\underline{\beta}}^{\bar{\beta}} U_{k}(\beta) f(\beta) d \beta}{\partial g}=0 .
$$

Using (9) and integrating by parts we obtain

$$
\int_{\underline{\beta}}^{\bar{\beta}} U_{k}(\beta) f(\beta) d \beta=-\int_{\underline{\beta}}^{\bar{\beta}}(1-F(\beta)) v_{\beta}(a(\beta), \beta) d \beta,
$$

so that (12) can be rewritten as

$$
\pi(1-\alpha)\left[-1+\int_{\underline{\beta}}^{\bar{\beta}} H^{\prime} f(\beta) d \beta\right]-\pi \alpha \int_{\underline{\beta}}^{\bar{\beta}}(1-F(\beta)) v_{a \beta} \frac{\partial a(\beta)}{\partial g} d \beta=0 .
$$

Consequently, the sign of $\partial a, / \partial g$ will determine how the level of care affects the caregivers' utility, with this effect being represented by the second term of the expression.

Consider first the case where $\alpha=0$, so that children have no weight in the social welfare function. In this case, using the parents' FOCs, (13) and (A.2) yields

$$
U^{\prime}(s)=\int_{\underline{\beta}}^{\bar{\beta}} H^{\prime} f(\beta) d \beta=1 .
$$

\footnotetext{
${ }^{10} \mathrm{We}$ assume that the second order condition holds.
} 
The optimal $g$ is set according to the same rule as under full information. While this ensures the same level of $s$, the levels of $m$ will generally be different under asymmetric information, and consequently the levels of the public LTC insurance benefit $\beta$. Use superscripts $f$ and as to refer to the solutions of the full information and asymmetric information problem. When $g=g^{f}, \int_{\underline{\beta}}^{\bar{\beta}} H^{\prime}\left(m^{a s}(\beta)\right) f(\beta) d \beta>1$, because $m^{a s}(\beta)<$ $m^{f}(\beta)$ (as $a$ is distorted downward and children receive a positive rent). Consequently, in the optimal asymmetric information allocation, $g^{a s}>g^{f}$ : the optimal level of LTC benefits is larger under asymmetric information than under full information. Intuitively, $g$ is higher to compensate in part for the downward distortion in $a$.

When $\alpha>0$, children have some weight in the social welfare function. In this case, the optimal LTC benefit is not only designed to provide insurance to parents. The government also takes into account the impact of public LTC insurance on the rents enjoyed by children. If $\partial a / \partial g>0$ increasing $g$ to provide more insurance to parents increases $a$, which also implies higher rents for children. If $\partial a / \partial g<0$, the two effects go in opposite directions.

The sign of $\partial a / \partial g$ is then crucial to characterize the optimal LTC policy. In the Appendix we prove the following lemma.

Lemma 1 Consider the case where parents do not observe the children's cost of providing care, and the government sets a uniform LTC benefit $g$ financed through a lumpsum payment by young parents. If the utility function in case of dependence, $H$ displays DARA (decreasing absolute risk aversion), and the solution is interior we have $\partial a / \partial g<0$.

Intuitively, with DARA, as $g$ increases, parents become less risk averse. Consequently, distorting down informal care, and thus consumption in the bad state of nature, is less costly for them.

Using the first-order condition of the parents with respect to $s,($ A.2), equation (13) implies that, if $\alpha>0$ and $H$ displays DARA

$$
U^{\prime}(s)<1<\int_{\underline{\beta}}^{\bar{\beta}} H^{\prime} f(\beta) d \beta .
$$


The utility of dependent parents is distorted down with respect to the full information case under DARA. Providing full insurance against the risk of dependence would push parents to cut the utility of low-cost children (by distorting down $\underline{a}$ ). From the perspective of social welfare, there is then a tradeoff between insurance and children's utility, leading to less than full insurance. Accordingly, parents have an incentive to save more and this increases their consumption if healthy.

Using equation (13) and Lemma 1 we can study the effect of $\alpha$ on $g^{a s}(\alpha)$. In the appendix we show that, under DARA

$$
\frac{\partial g}{\partial \alpha}<0
$$

In words, $g$ decreases in the weight of children in the social welfare function. The optimal uniform transfer as a function of the weight of children in the social welfare function is represented in Figure 1. When $\alpha=1$, only the rent of the children enters in the social welfare function, and the government sets the transfer $g$ equal to zero.

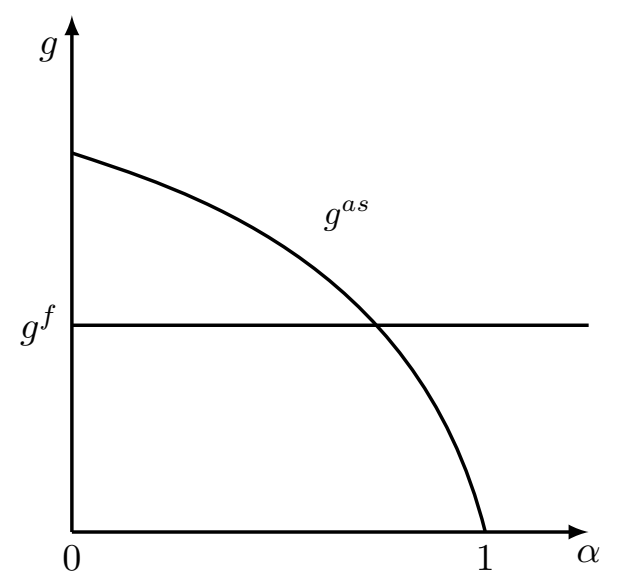

Figure 1: Uniform LTC transfer under symmetric and asymmetric information

To sum up, $g$ decreases in $\alpha$, while for all $\beta<\bar{\beta}$ informal care $a(\beta)$ (chosen by parents) is decreasing in $g .{ }^{11}$ Consequently, $a(\beta)$ increases in $\alpha$, which is the children's weight in social welfare. This implies that the larger the weight of children in the social

\footnotetext{
${ }^{11}$ Parents' choices do not directly depend on $\alpha$.
} 
welfare function, the larger the optimal average level of informal care. This apparently counter-intuitive result is due to the exchange motive behind informal care. Under this motive, a higher reliance on the family for the provision of long-term care implies higher rents for children. The optimal policy implies that children will provide more informal care than in the laissez-faire, for which they will be compensated by higher transfers. Family help under the optimal uniform LTC policy is represented in Figure 2. The distortion in $a(\beta)$ in the asymmetric information is mitigated by the government policy. The higher the weight of children in the social welfare function, the smaller the distortion. However, no matter how large $\alpha$ is, the distortion in $a(\beta)$ does not disappear. To see this, recall that equation (11) implies that $v_{a}(a(\beta,) \beta)$ is always strictly smaller than 1 for all $\beta<\bar{\beta}$.

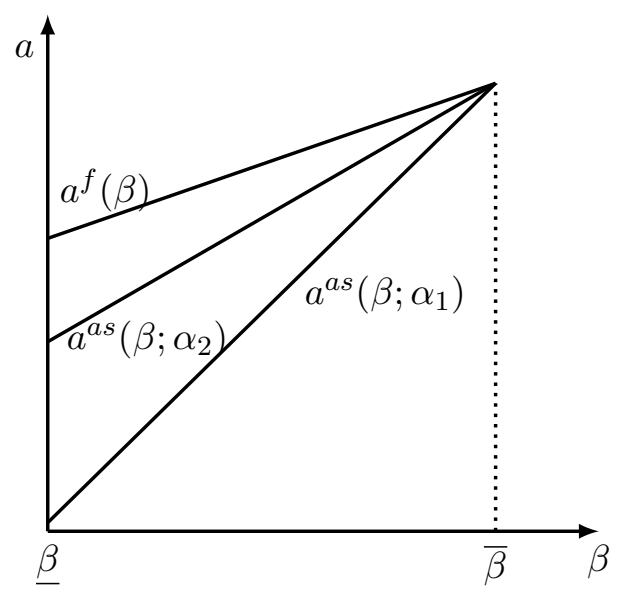

Figure 2: Family help as a function of $\beta$ under symmetric and asymmetric information, with $\alpha_{1}<\alpha_{2}$.

The results obtained in this section are summarized in the following proposition.

Proposition 1 Consider the case where the children's cost of providing care is not observable and where public policy is restricted to a uniform LTC benefit $g$ financed by a lump-sum tax. Informal care is observable only by parents. Assuming an interior solution, the optimal LTC policy is such that:

(i) The risk of having children with a high cost of providing care is not fully insured; 
(ii) If children's utility has no weight in social welfare, parents are fully insured against dependence. This is achieved through a uniform benefit that is larger than in the full information case;

(iii) If the weight of children in social welfare is strictly positive, and the parents' utility in case of dependence $H(m)$ exhibits DARA, parents are less than fully insured against dependence. The uniform benefit decreases with the weight of children in social welfare; and

(v) The average level of care provided by children increases with their weight in social welfare.

Up to now, we have assumed an interior solution for both $a^{f}$ and $a^{a s}$, however, corner solutions are possible. Assuming that $a$ is interior at least for the lowest-cost children $(\bar{\beta})$, two types of corner solutions may arise. First, we can have the case where $a^{f}$ is always interior but where $a^{a s}=0$ for all $\beta$ below some benchmark $\beta_{0}$. Second, we can have that both $a^{f}$ and $a^{a s}$ are zero for low $\beta$ 's (high-cost children), where the critical benchmark is smaller under full than under asymmetric information (due to the downward distortion). ${ }^{12}$ The corner solutions are depicted in Figure 3.

\section{Nonlinear policies}

We now consider nonlinear policies where bequests are publicly observable and social care $g$ can be conditioned on the transfer, $\tau$. The LTC policy can then screen for the level of children's cost parameter $\beta$. We continue to assume that $a$ is observable only to parents. The underlying problem presents methodological challenges because we have to deal with a "nested" principal-agent problem. ${ }^{13}$ While the policy can screen for $\beta$ this is done only indirectly via the parents. These do not observe their child's $\beta$ either but since they observe informal care, they have superior information.

\footnotetext{
${ }^{12}$ The first case occurs when $v_{a}(0, \beta)<1 \forall \beta$ and $1-v_{a}(0, \beta)+v_{a \beta}(0, \beta) \int_{\beta}^{\bar{\beta}} H^{\prime} f(s) d s / H^{\prime} f(\beta)<0$ for $\beta<\beta_{0}<\bar{\beta}$. The second one arises when $v_{a}(0, \beta)>1$ for $\beta<\widehat{\beta}<\bar{\beta}$.

${ }^{13}$ The problem considered by Guesnerie and Laffont (1978) has a similar structure. They analyze nonlinear taxation of a monopolist who itself uses nonlinear pricing.
} 

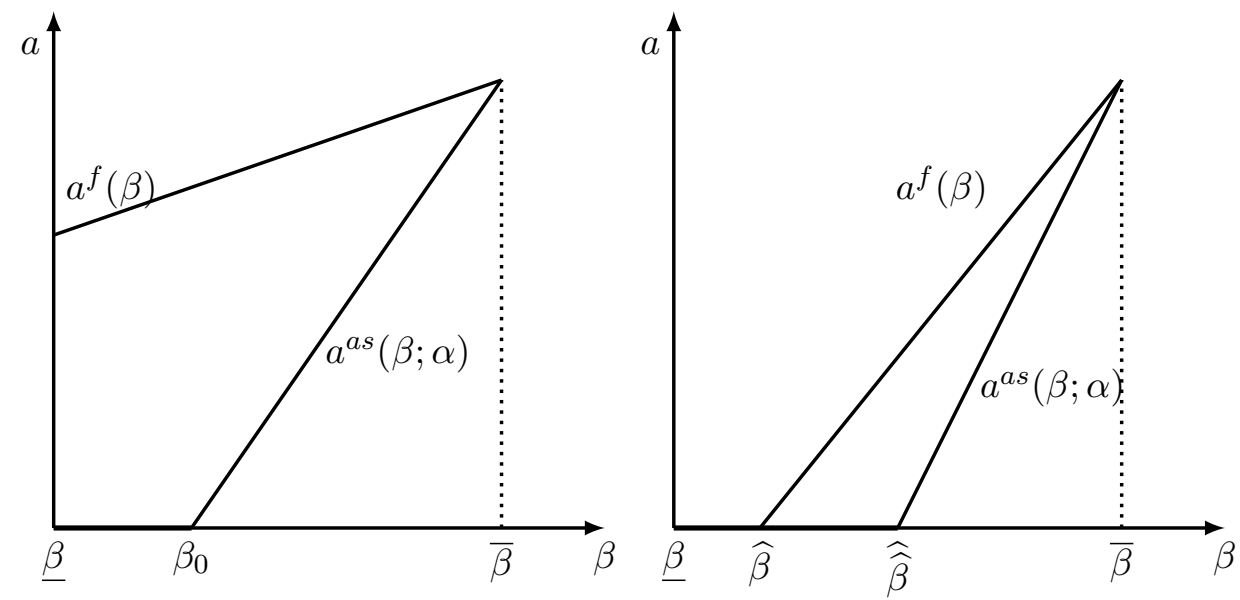

Figure 3: Family help as a function of $\beta$ under symmetric and asymmetric information, corner solutions.

We proceed exactly like as in the previous section. We start with the full information solution, then we consider the case where only parents have full information (about their children). Finally, we turn to the most interesting case, where neither the parents nor the government can observe the children's type $\beta$. Parents observe $a$ but the government does not. The policy we study consists of a menu of LTC benefits and bequests. Under asymmetric information parents self-select into a benefit-bequest pair. We continue to assume that the government cannot make any direct transfer to children.

Observe that while we study a mechanism design problem, the policy can be implemented by a suitably designed LTC and tax policy. How this can be done depends on the precise timing and, more specifically, on whether $\tau$ is interpreted as a gift or a bequest. When $\tau$ is a gift and thus precedes the public transfer $g$, the solution can be implemented simply by a function $g(\tau)$ conditioning LTC benefits on $\tau$. When $\tau$ is a bequest, which by definition occurs after $g$ is consumed, we can condition LTC on a reported (planned) $\tau$, but then we have to make sure that parents remain committed to one contract in the menu $(\tau, g)$. In particular we have to prevent parents from leaving a larger bequest than specified in the chosen contract (to "buy" more care). This can be done by a nonlinear tax on transfers, which is prohibitively large when $\tau$ deviates from that associated with the level of public care consumed. In practice, this means that 
"excess" public care can be recuperated from an individual's bequest. In other words, social LTC policy includes the possibility of so-called "estate recovery"; see Courbage and Montoliu-Montes (2020).

\subsection{Full information benchmark}

Assume for the time being that both parents and the government have full information and observe the cost parameter of children. The government sets a menu of contracts $(\tau(\beta), g(\beta))$. Parents will set $a(\beta)$ such that $\tau(\beta)=v(\alpha(\beta), \beta)$, which ensures the participation constraint is just satisfied for all children, and choose $s$ ex ante to maximize their expected utility. Consequently, their behavior directly follows from the policy. Under full information, the binding participation constraint continues to ensure that the rent of the children is equal to zero and does not appear in social welfare. The government's problem is

$$
\begin{aligned}
\max _{g(\beta)} \quad G^{f n}= & w \bar{T}-\pi \int_{\underline{\beta}}^{\bar{\beta}} g(\beta) f(\beta) d \beta-s+(1-\pi) U(s) \\
& +\pi \int_{\underline{\beta}}^{\bar{\beta}} H(s+a-v(a, \beta)+g(\beta)) f(\beta) d \beta,
\end{aligned}
$$

where $s$ and $a$ are the solutions to the parents' problem, and the superscript $f n$ denotes full information and nonlinear policies. Under full information, the levels of $a$ and $\tau$ do not depend on $g(\beta)$ and are given by (5) and the children's participation constraints.

Using the envelope theorem, the first-order condition of the government is ${ }^{14}$

$$
\frac{\partial G^{f n}}{\partial g(\beta)}=-\pi f(\beta)+\pi H^{\prime}(s+a-v(a, \beta)+g(\beta)) f(\beta)=0 .
$$

Combining this condition with (4) yields

$$
U^{\prime}(s)=H^{\prime}(s+a-v(a, \beta)+g(\beta))=1,
$$

implying that the marginal utility of consumption must be equalized across time, dependence states, and children's types. The government is now able to equalize consumption

\footnotetext{
${ }^{14}$ We assume that the second order condition holds.
} 
across parents with different types of children which was never possible with a uniform policy. For the rest, with full information and unrestricted instruments it is not surprising that that solution involves full insurance.

\subsection{Asymmetric information}

Consider now the case where neither the parents nor the government can observe the children's type. The government designs a schedule $(g, \tau)$. As in the full information case, the parents' behavior then directly follows from the policy. Specifically, the parents set a schedule $(a, \tau)$, taking into account the government's policy and the children's incentive and participation constraints. The children's utility is then given by equation (9).

The problem of the government is now given by

$$
\begin{aligned}
\max _{g(\beta)} \quad G^{a s n}= & (1-\alpha)\left[w \bar{T}-\pi \int_{\underline{\beta}}^{\bar{\beta}} g(\beta) f(\beta) d \beta-s+(1-\pi) U(s)\right] \\
& +(1-\alpha)\left[\pi \int_{\underline{\beta}}^{\bar{\beta}} H\left(s+a-v(a, \beta)-U_{k}(\beta)+g(\beta)\right) f(\beta) d \beta\right] \\
& +\alpha \pi \int_{\underline{\beta}}^{\bar{\beta}} U_{k}(\beta) f(\beta) d \beta \\
\text { s.t. } \quad & U_{k}(\beta)=-\int_{\underline{\beta}}^{\beta} v_{\beta} d x .
\end{aligned}
$$

We assume that $a^{\prime}(\beta)>0$ holds, so that the second order condition for children's incentive compatibility is satisfied, and that the contract is separating.

We show in Appendix A.5 that the optimal levels of informal care satisfy the following condition:

$$
1-v_{a}(a(\beta,) \beta)=-\frac{1-F(\beta)}{f(\beta)} \frac{(1-2 \alpha)}{(1-\alpha)} v_{a \beta} .
$$

This condition implies that $a(\beta)$ is distorted with respect to the first best for all $\beta<\bar{\beta}$. The direction of the distortion depends on the weight of children in the social welfare function. If $\alpha<1 / 2$, then informal care is distorted down. If $\alpha>1 / 2$, there is an upward distortion. In this case, we may have a situation where the optimal contract implies some pooling, as $a^{\prime}(\beta)>0$ may not hold. We rule out this possibility and focus on separating contracts. 
As in the case of the uniform policy, under a nonlinear policy informal care also increases as the weight of children in the social welfare function increases.

The optimal public LTC transfer is set in relation to the consumption of dependent parents $m(\beta)$ across children's types. Imposing that $m(\beta)=a(\beta)-\tau(\beta)+g(\beta)$ is constant implies that $-a^{\prime}(\beta)+\tau^{\prime}(\beta)=g^{\prime}(\beta)$. Since $\tau(\beta)=v(a(\beta), \beta)-\int_{\underline{\beta}}^{\beta} v_{\beta} d x$, then $g^{\prime}(\beta)=-a^{\prime}(\beta)+\tau^{\prime}(\beta)=-\left(1-v_{a}\right) a^{\prime}(\beta)+v_{a \beta}+v_{\beta}$. Under our assumptions that $v_{a \beta}<0$ and $v_{\beta}<0$, and the incentive compatibility condition $a^{\prime}(\beta)>0$, we have that $g^{\prime}<0$ whenever $1-v_{a}>0$, which is always true if $\alpha<1 / 2$.

The results obtained in this section are summarized in the following proposition.

Proposition 2 Consider the case where the children's cost of providing care is not observable and where the LTC benefit $g$, financed by a lump sum tax, can be conditioned on the transfer $\tau$. Informal care is observable only by parents. Assuming that the schedule $(a, \tau)$ offered to children is separating, the optimal LTC policy is such that:

(i) The risk of having children with a high cost of providing care is fully insured;

(ii) If the weight of children in the social welfare function, $\alpha$, is smaller than half, then informal care is distorted down. If $\alpha$ is greater than half, then informal care is distorted up; and

(iii) The average level of care provided by children increases with their weight in social welfare.

\section{Calibrated illustration}

In this section, we simulate our model using a calibrated example.

\subsection{Calibration}

We assume that $v(a, \beta)=a^{2} / 2 \beta$. Norton et al. (2013) estimate the elasticity of informal care (expressed in hours per year) and financial transfers to children at 0.251 . This would correspond to $a \tau^{\prime}(a) / \tau(a)$ in the notation of our model. Using the fact that, for the latest year in their study, the average number of hours of informal care is equal to 395 and the average transfer (conditional on any transfer at all) is equal to 4,669 USD, we 
extrapolate an average $\tau^{\prime}(a)=2.97$. Solving the child's problem, we have $v_{a}=\tau^{\prime}(a)$, which for the average child reduces to $395 / \beta=2.97$. This yields an average value of $\beta$ of 133. Under the assumption that $\beta$ is uniformly distributed over $[0, \bar{\beta}]$, such an average implies that the upper bound of $\beta$ is equal to 266 .

We set the level of lifetime savings at 60,000 USD (see De Nardi et al., 2010, who find that this was the 1996 median assets of individuals aged 74 in the US). We also assume that dependence lasts for 5 years, but this is simply for the sake of illustration and has no impact on the results.

To simplify the computations, we consider the following parents' utility, which is linear in a composite consumption good and concave in care

$$
\begin{aligned}
& w \bar{T}-s+(1-\pi) s+\pi s \\
& -5 \pi \int_{0}^{\bar{\beta}} \frac{\tau(\beta)+p(z(\beta)+g(\beta))}{\bar{\beta}} d \beta+5 \pi \int_{0}^{\bar{\beta}} \frac{\gamma \ln (a(\beta)+z(\beta)+g(\beta))}{\bar{\beta}} d \beta,
\end{aligned}
$$

where $\tau(\beta)=v(a(\beta), \beta)+U_{k}(\beta)=a(\beta)^{2} / 2 \beta+\int_{0}^{\beta} a(x)^{2} / 2 x^{2} d x$. Care is the sum of informal care, $a(\beta)$, formal care purchased on the market, $z(\beta)$, and the care provided by public insurance, $g$, which is flat under the uniform policy, and depends on $\beta$ in the nonlinear case. Formal care has a market price equal to $p$.

Integrating by parts the term containing $\tau(\beta)$, we obtain the following expression for the utility of the parents

$$
\begin{aligned}
& w \bar{T}-5 \pi \int_{0}^{\bar{\beta}}(1-F(\beta)) \frac{a(\beta)^{2}}{2 \beta^{2}} d \beta-5 \pi \int_{0}^{\bar{\beta}} \frac{1}{\bar{\beta}} \frac{a(\beta)^{2}}{2 \beta} d \beta \\
& -5 \pi \int_{0}^{\bar{\beta}} \frac{p(z(\beta)+g(\beta))}{\bar{\beta}} d \beta+5 \pi \int_{0}^{\bar{\beta}} \frac{\gamma \ln (a(\beta)+z(\beta)+g(\beta))}{\bar{\beta}} d \beta,
\end{aligned}
$$

Consider first the case without any public insurance. For an interior solution, the first-order condition for the parents with respect to formal and informal care yield, respectively:

$$
\frac{\gamma}{z(\beta)+a(\beta)}=p
$$

and

$$
\frac{\gamma}{\bar{\beta}(z(\beta)+a(\beta))}-\frac{a(\beta)}{\bar{\beta} \beta}-\frac{(\bar{\beta}-\beta) a(\beta)}{\bar{\beta} \beta^{2}}=0 .
$$


Substituting (16) in (17), we obtain

$$
a(\beta)=\frac{p \beta^{2}}{\bar{\beta}} .
$$

We set the price of formal care $p$ at 7.25 USD, which is the federal minimum wage in the US in 2020. We focus here on formal home care, as opposed to nursing home care. The former is often provided by low-skilled workers receiving a low compensation. This implies that the optimal contract set up by the parents stipulates $a(\beta)=0.027 \beta^{2}$. Children with $\beta=0$ should not provide any care, while children with the average cost parameter (133) should deliver 477 hours of care per year (for comparison, the average informal care documented by Norton et al., 2013, is 395 hours per year).

Concerning the level of formal care, we calibrate the parameter $\gamma$ so that, on average, formal care represents $13 \%$ of total care, or equivalently, $15 \%$ of informal care. This corresponds to the share of formal home-care reported by Barczyk and Kredler (2018). ${ }^{15}$ For a family providing the average level of informal care $(a=395)$, this implies that $\gamma=3,975$.

We have corner solutions such that $z=0$ whenever $a>548$ hours per year, which occurs if $\beta>143$. In this case, the optimal $a$ is given by

$$
\frac{\gamma}{\bar{\beta} a(\beta)}-\frac{a(\beta)}{\bar{\beta} \beta}-\frac{(\bar{\beta}-\beta) a(\beta)}{\bar{\beta} \beta^{2}}=0 .
$$

Solving for $a$ and rearranging yields

$$
a(\beta)=\frac{\sqrt{\gamma} \beta}{\sqrt{266}}=3.87 \beta
$$

According to this expression, children with the lowest $\operatorname{cost}(\beta=\bar{\beta})$ should provide 1028 hours of informal care per year.

\subsection{Uniform policy}

With the specific utility function considered in this section, savings can be freely allocated between formal care and other consumption. Formal care is chosen once $\beta$ is known while a uniform $g$ is, by definition, the same in all states of nature. Consequently,

\footnotetext{
${ }^{15}$ Again, we focus on home care and we exclude individuals receiving nursing home care.
} 
it is plain that formal private care $z$ dominates the uniform $g$. Consequently, under the specification used in the calibration, when private formal care is available there is no room for $g$.

To provide a more telling illustration of our analytical results we thus assume for the remainder of this subsection that there is no private market for formal care so that $z=0$.

In this case, for all $\beta, a(\beta, g)$ is defined as in (18). The highest cost children provide no informal care, while the average level of informal care is 514 hours per year. The problem of the government is

$$
\begin{aligned}
& \max _{g}-(1-\alpha) \pi \int_{0}^{\bar{\beta}} \frac{p g}{\bar{\beta}} d \beta \\
& -(1-\alpha) \pi\left(\int_{0}^{\bar{\beta}}(1-F(\beta)) \frac{a(\beta)^{2}}{2 \beta^{2}} d \beta+\int_{0}^{\bar{\beta}} \frac{1}{\bar{\beta}} \frac{a(\beta)^{2}}{2 \beta} d \beta\right) \\
& +(1-\alpha) \pi \int_{0}^{\bar{\beta}} \frac{\gamma \ln (a(\beta, g)+g)}{\bar{\beta}} d \beta+\alpha \pi \int_{0}^{\bar{\beta}} \frac{\bar{\beta}-\beta}{\bar{\beta}} \frac{a(\beta, g)^{2}}{2 \beta^{2}} d \beta .
\end{aligned}
$$

The first-order condition, using the envelope theorem, is given by

$$
(1-\alpha) \pi \int_{0}^{\bar{\beta}}\left(\frac{\gamma}{a+g}-p\right) d \beta-\alpha \pi \int_{0}^{\bar{\beta}}(\bar{\beta}-\beta) \frac{1}{\beta^{2}} \frac{a^{2}}{2 a+g} d \beta=0 .
$$

Table 1 illustrates the uniform policy and the implied profile of informal care for different values of $\alpha$. If children are not included in the social welfare function $(\alpha=0)$, the optimal public LTC transfer is equal to 262, which corresponds to the level providing full insurance against the risk of dependence. As the weight of children increases, the public LTC transfer decreases, while the level of informal care for each $\beta$ increases. Public care crowds out informal care, which under the uniform policy is lower than the laissez-faire informal care for all $\beta$.

Overall, the optimal share of public LTC in total care (evaluated for the average parent) should range between $40 \%$ when $\alpha=0$ and $29 \%$ when $\alpha=0.5$. Social welfare decreases in $\alpha$ in both the laissez faire and under the optimal uniform policy. ${ }^{16}$ More

\footnotetext{
${ }^{16}$ We mention this purely for the record because the underlying comparison is debatable. In any event
} 


\begin{tabular}{l|r|rrrrr}
\hline & LF & $\alpha=0$ & $\alpha=0.10$ & $\alpha=0.25$ & $\alpha=0.40$ & $\alpha=0.50$ \\
\hline$g$ & 0 & 262 & 254 & 236 & 208 & 175 \\
\hline$a(0)$ & 0 & 0 & 0 & 0 & 0 & 0 \\
$a(50)$ & 194 & 103 & 105 & 109 & 116 & 125 \\
$a(100)$ & 387 & 278 & 281 & 287 & 297 & 309 \\
$a(133)$ & 515 & 401 & 404 & 410 & 422 & 435 \\
$a(160)$ & 619 & 502 & 506 & 513 & 525 & 538 \\
$a(266)$ & 1,029 & 908 & 911 & 918 & 932 & 946 \\
\hline$g /\left.(g+a)\right|_{\beta=133}$ & $0 \%$ & $40 \%$ & $39 \%$ & $37 \%$ & $33 \%$ & $29 \%$ \\
Social welfare in LF & & 21,606 & 19,546 & 16,454 & 13,362 & 11,301 \\
Social welfare uniform policy & & 22,559 & 20,343 & 17,021 & 13,704 & 11,498 \\
$\Delta$ welfare uniform policy & & $+4.4 \%$ & $+4.1 \%$ & $+3.4 \%$ & $+2.6 \%$ & $+1.7 \%$ \\
\hline
\end{tabular}

Table 1: Numerical illustrations of the uniform policy

interestingly, the welfare improvement obtained by means of the uniform policy over the laissez-faire depends on the children's weight in the social welfare function, $\alpha$. The higher this weight, the lower the welfare improvement (in percentage points) that can be obtained by the uniform policy. Intuitively this reflects that fact that $g$ is most powerful when we care only about parents; when children enter the SWF the optimal $g$ results from a tradeoff because it decreases children's utilities.

\subsection{Nonlinear policy}

With a nonlinear transfer, the Hamiltonian corresponding to the problem of the government, which in the general case is given in (A.8), can be rewritten as

$$
\begin{aligned}
\mathcal{H} & =(1-\alpha)[w \bar{T}-\pi g(\beta) f(\beta)] \\
& (1-\alpha)\left[-5 \pi \frac{\tau(\beta)+p(z(\beta)+g(\beta))}{\bar{\beta}}+5 \pi \frac{\gamma \ln (a(\beta)+z(\beta)+g(\beta))}{\bar{\beta}}\right] \\
& +5 \alpha \pi \int_{0}^{\beta} \frac{a(x)^{2}}{2 \bar{\beta} x^{2}} d x-\mu_{\beta} \frac{a(\beta)}{2 \beta^{2}} .
\end{aligned}
$$

it is not a robust result because it depends on the relative scaling of parents' and children's utilities (of which we take a weighted average) which is arbitrary. In particular, adding a sufficiently large constant to children's utility would reverse this property while not affecting the other results. 
The first-order condition with respect to informal care is now given by

$$
\frac{\gamma}{a+g+z}-\frac{a}{\beta}=\frac{1-2 \alpha}{1-\alpha}(\bar{\beta}-\beta) \frac{a}{\beta^{2}} .
$$

Furthermore, the first-order condition of the government's problem with respect to $g(\beta)$ is

$$
\frac{\gamma}{(a+z+g)}=p
$$

This is equivalent to the first-order condition of the parents with respect to $z$. Consequently, $g$ and $z$ are redundant instruments, and to concentrate on $g$ we assume in remainder of the section that $z=0$. Unlike in the uniform case considered in the previous subsection, $g$ can now also be adjusted according to $\beta$ so that $g(\beta)$ and $z(\beta)$ are perfectly equivalent. Substituting (20) into (19), we obtain

$$
a(\beta)=\frac{p \beta^{2}}{\frac{1-2 \alpha}{1-\alpha}(\bar{\beta}-\beta)+\beta}=\frac{7.25(1-\alpha) \beta^{2}}{\bar{\beta}(1-2 \alpha)+\alpha \beta} .
$$

Differentiating this expression with respect to $\beta$ yields

$$
\frac{\partial a}{\partial \beta}=\frac{(14.5) \bar{\beta}(1-\alpha) \beta(1-2 \alpha)}{(\bar{\beta}(1-2 \alpha)+\alpha \beta)^{2}},
$$

which is positive if and only if $\alpha \leq 1 / 2$. This is in line with the result obtained in the analytical part where we have shown that for $\alpha>1 / 2$ the solution may involve pooling. We shall continue to concentrate on this range of $\alpha$ 's. Simple inspection of (21) shows that $a(\beta)$ is positive as long as $1-2 \alpha>0$, while differentiating the expression shows that $a$ is strictly increasing in $\alpha$ for all types except the lowest-cost children. ${ }^{17}$

Table 2 illustrates the resulting informal care, transfers to children, and government transfers for different levels of $\beta(0,50,100,133,160$ and 266). We find that the total level of care is constant and equal to 548 hours per year, which is consistent with $(20)^{18}$. Informal care increases in $\beta$, as well as $\tau$. Conversely, the government transfer decreases in $\beta$. As long as public care is positive, it increases with the children's cost of providing

\footnotetext{
${ }^{17}$ We have

$$
\frac{\partial a}{\partial \alpha}=7.25 \beta^{2} \frac{\bar{\beta}-\beta}{(\bar{\beta}-2 \bar{\beta} \alpha+\alpha \beta)^{2}},
$$

which is strictly positive for all $\beta<\bar{\beta}$.

${ }^{18}$ According to $(20)$, total care is equal to $\gamma / p=3,975 / 7.25=548$.
} 
care (decreases with $\beta$ ) at a rate that is larger, the larger the weight of children in social welfare. For high levels of $\beta$, we have corner solutions for $g(\beta)$, such that $g(\beta)=0$ for all $\beta>\widetilde{\beta}$, where $\widetilde{\beta}$ is such that $a(\widetilde{\beta})=\gamma / p$. Since, for all $\beta, a(\beta)$ is higher the higher the weight of children in social welfare, the threshold $\widetilde{\beta}$ decreases in $\alpha$. In the corner solutions, there is pooling in the level of informal care: $a(\beta)=a(\widetilde{\beta})$ and $\tau(\beta)=\tau(\widetilde{\beta})$ for all $\beta>\widetilde{\beta}$.

Under the optimal nonlinear policy, the share of public care on total care for the average individual decreases with the weight of children in social welfare and should range between $12 \%$ and $0 \%$. Not surprisingly, social welfare is always higher than under the uniform policy and yields higher welfare improvements with respect to the laissez faire. The nonlinear policy allows us to target parents whose children have a high cost of providing informal care, thus providing insurance against the risk associated with the variance in informal care.

The optimal levels of informal care, transfers to children, and government transfers are depicted in Figures 4-6 as a function of the cost parameter (for $\alpha=0.25$ ). In this case the threshold $\widetilde{\beta}$ (above which one has corner solutions) is equal to 129 . As already presented in Table 2, informal care and transfers to children increase in $\beta$, while the optimal public transfer decreases in the cost parameter.

In Figure 7, we plotted the optimal level of the public LTC transfer as a function of the level of intrafamily transfers $\tau$, for different levels of $\alpha$. As already illustrated in Table 2, $g(\tau)$ is a decreasing function of $\tau$. This function is steeper for lower levels of $\alpha$. Intuitively, the government needs to provide insurance for the parents. As a higher $\tau$ is associate with higher levels of informal care, $g$ decreases in $\tau$. At the same time, the government also wants to ensure that parents elicit (and pay) a sufficient amount of informal care from their children. To this end, $g$ should not decrease "too fast" in $\tau$. Since the latter government objective is more important for higher $\alpha$, then the function $g(\tau)$ is flatter when the weight of children in social welfare is higher. 


\begin{tabular}{rrrrrr}
\hline & $\alpha=0$ & $\alpha=0.10$ & $\alpha=0.25$ & $\alpha=0.40$ & $\alpha=0.50$ \\
\hline$(0)$ & 0 & 0 & 0 & 0 & 0 \\
$g(0)$ & 0 & 0 & 0 & 0 & 0 \\
$a(50)$ & 648 & 548 & 548 & 548 & 548 \\
$\tau(50)$ & 62 & 75 & 93 & 148 & 362 \\
$g(50)$ & 480 & 473 & 455 & 400 & 186 \\
\hline$a(100)$ & 273 & 293 & 344 & 467 & 548 \\
$\tau(100)$ & 495 & 575 & 807 & 1,559 & 3,975 \\
$g(100)$ & 276 & 255 & 204 & 81 & 0 \\
\hline$a(133)$ & 482 & 510 & 548 & 548 & 548 \\
$\tau(133)$ & 1,165 & 1,316 & 1,595 & 1,951 & 3,975 \\
$g(133)$ & 66 & 38 & 0 & 0 & 0 \\
\hline$a(160)$ & 548 & 548 & 548 & 548 & 548 \\
$\tau(160)$ & 1,413 & 1,464 & 1,595 & 1,951 & 3,975 \\
$g(160)$ & 0 & 0 & 0 & 0 & 0 \\
\hline$a(266)$ & 548 & 548 & 548 & 548 & 548 \\
$\tau(266)$ & 1,413 & 1,464 & 1,595 & 1,951 & 3,975 \\
$g(266)$ & 0 & 0 & 0 & 0 & 0 \\
\hline$\widetilde{\beta}$ & 142 & 138 & 129 & 111 & 76 \\
\hline Social welfare in LF & 21,606 & 19,546 & 16,454 & 13,362 & 11,301 \\
\hline Social welfare nonlinear policy & 22,643 & 20,420 & 17,096 & 13,811 & 11,756 \\
\hline welfare nonlinear policy & $+4.8 \%$ & $+4.5 \%$ & $+3.9 \%$ & $+3.4 \%$ & $+4.0 \%$ \\
\hline & & & & & $0 \%$ \\
\hline
\end{tabular}

Table 2: Numerical illustrations of the nonlinear policy 

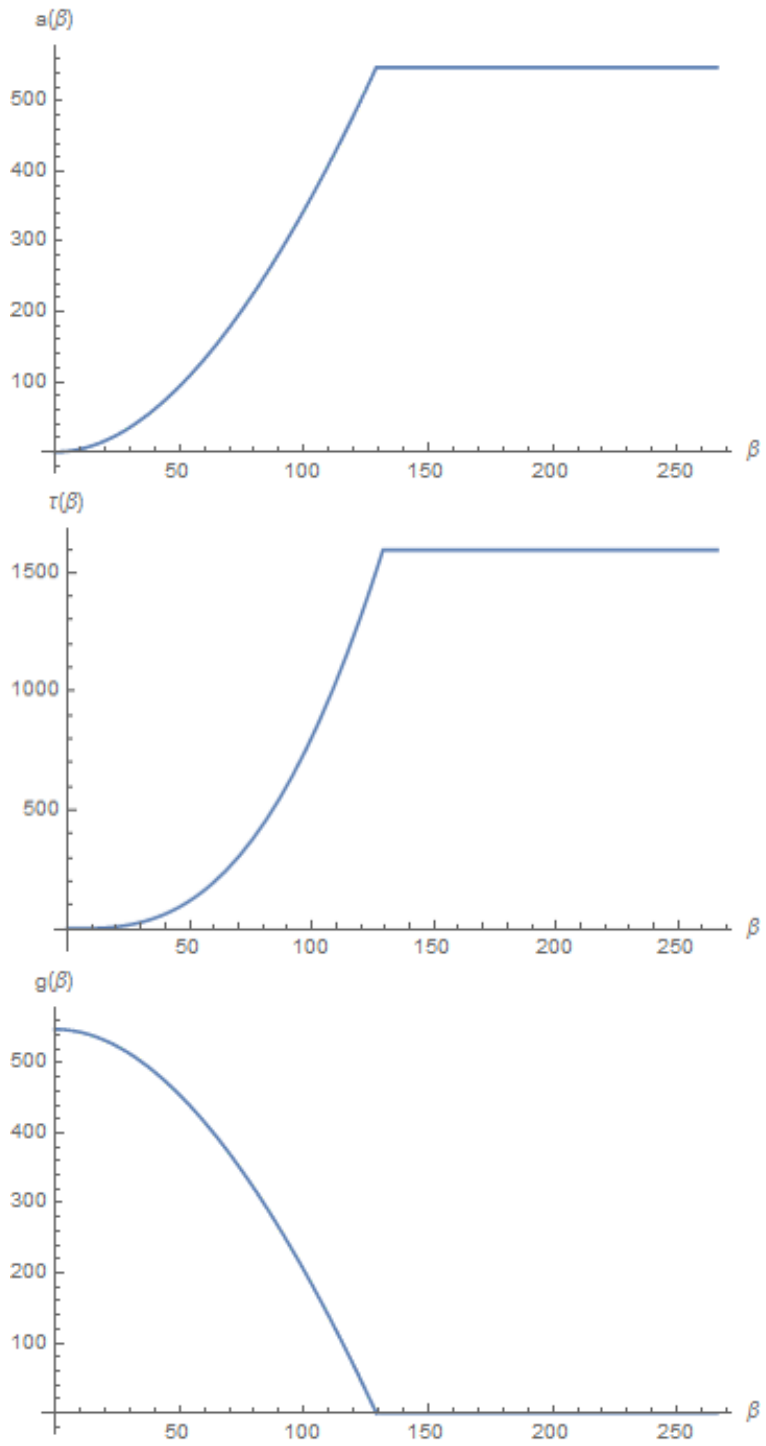

Figure 4: Nonlinear policy: optimal levels of informal care, family transfer, and public LTC transfers as a function of children's cost parameter, for $\alpha=0.25$. 


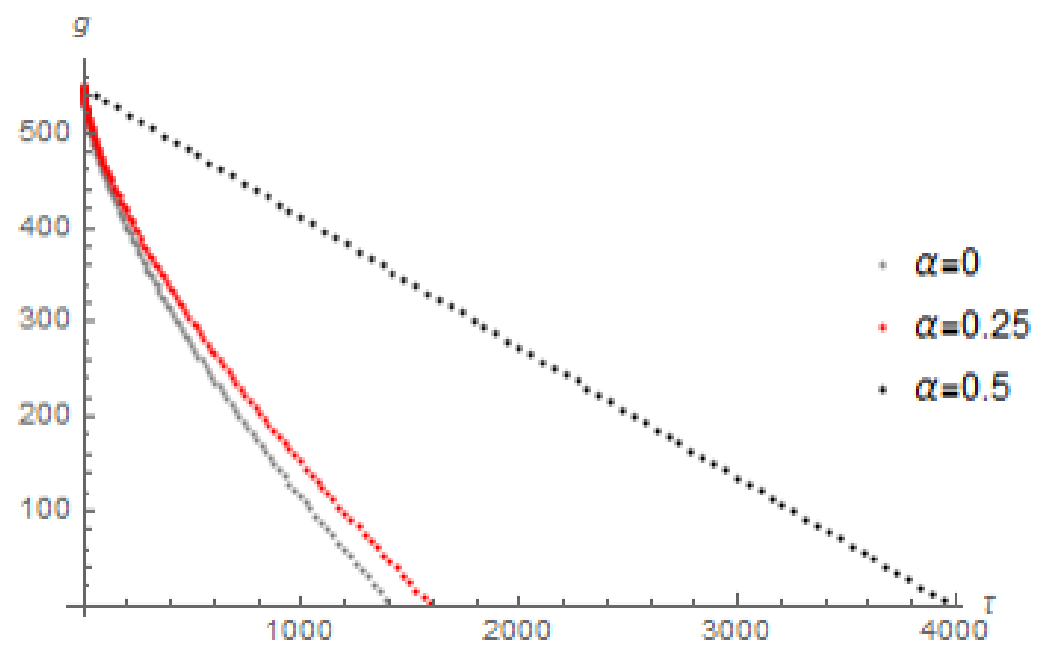

Figure 5: Non-uniform policy implementation for $\alpha=0, \alpha=0.25$, and $\alpha=0.5$.

\section{Summary and concluding comments}

We have studied the design of social LTC insurance when informal care is exchangebased. Parents do not observe their children's cost of providing informal care but they can commit to a rule specifying transfers that are conditional on the level of informal care. Social LTC insurance is designed to maximize a weighted sum of parents' and children's utility. The optimal uniform public LTC insurance can insure the risk of dependence but when children's utility matters, parents are not fully insured. A nonlinear policy conditioning LTC benefits on bequests provides full insurance, even for the risk of having children with a high cost of providing care. Under the optimal public policy, informal care always increases in the children's welfare weight, irrespective of the parents' degree of risk aversion.

The numerical solutions based on a calibrated example illustrate the empirical implication of our analysis. In the uniform case, public care should represent about $40 \%$ of total care when SWF considers only the parents' utility. The share of public care decreases to about 30 as the weight of children increase. In the nonlinear case, public care increases with the children's cost of providing care, at a rate that is larger, the larger the weight of children in social welfare. It represents, on average, about one-tenth of 
total care when the weight of children in SWF is zero: it decreases with the weight of children.

Our example is based on a very rough estimation of the distribution of the children's cost parameter, which must be followed up by a more sophisticated estimation of this and other ingredients of our model. These limitations notwithstanding, this illustrates the potential of our theoretical framework to provide a basis for empirical analysis of the underlying problem. 


\section{References}

[1] Alessie, R., V. Angelini and G. Pasini, (2014), "Is it true love? Altruism versus exchange in time and money transfers ", De Economist, 162, 193-213.

[2] Arrondel, L. and A. Masson, (2006), "Altruism, exchange or indirect reciprocity: what do the data on family transfers show?", In: Kolm, S.C. and J. Mercier-Ytier (Eds.), Handbook of the Economics of Giving, Altruism and Reciprocity, Volume 2, North Holland, Chapter 14.

[3] Barczyk D. and M. Kredler, (2018), "Evaluating long-term-care policy options, taking the family seriously", Review of Economic Studies, 85, 766-809. 93(6), 10451076.

[4] Bernheim, B., A. Shleifer and L. Summers (1985), "The strategic bequest motive", Journal of Political Economy, 93(6), 1045-1076.

[5] Bolin, K., B. Lindgren and P. Lundborg, (2008), "Your next of kin or your own career? Caring and working among the 50+ of Europe", Journal of Health Economics, 27,718 "738.

[6] Bonsang, E. and J. Schoenmaeckers, (2015), "Long-term care insurance and the family: does the availability of potential caregivers substitute for long-term care insurance?", In: Börsch-Supan, A., T. Kneip and H. Litwin (Eds.), Ageing in Europe - Supporting Policies for an Inclusive Society, De Gruyter, Chapter 33.

[7] Brodaty, H. and M. Donkin, (2009), "Family caregivers of people with dementia", Dialogues in Clinical Neuroscience, 11, 217-228.

[8] Canta, C., H. Cremer and F. Gahvari, (2016), "Maybe 'honor thy father and thy mother': uncertain family aid and the design of social long term care insurance", TSE Working Paper 16-685.

[9] Canta, C. and H. Cremer, (2020), "Opting out and topping up reconsidered: informal care under uncertain altruism", Canadian Journal of Economics, forthcoming. 
[10] Canta, C. and H. Cremer, (2019), "Long-term care policy with nonlinear strategic bequests ", European Economic Review, 2019, 119, 548-566.

[11] Casamatta, G., Cremer, H. and Pestieau, P. (2000b) "Political sustainability and the design of social insurance", Journal of Public Economics, 75, 315-340.

[12] Ciani, E. and C. Deiana, (2018), "No free lunch, Buddy: past housing transfers and informal care later in life", Review of Economics of the Household, 16, 971-1001.

[13] Colombo, F., A. Llena-Nozal, J. Mercier and F. Tjadens, (2011), "Help Wanted? Providing and paying for long-term care", OECD Health Policy Studies, OECD Publishing.

[14] Coe, N.B. and C.H. Van Houtven, (2009), "Caring for Mom and Neglecting Yourself? The Health Effects of Caring for an Elderly Parent", Health Economics, 18, 991-1010.

[15] Courbage, C. and G. Montulio-Montes, (2020), "Estate recovery and long-term care insurance", Journal of Public Economic Theory, 22, 949-72.

[16] Cox, D. and M.R. Rank, (1992), "Inter-vivos transfers and intergenerational exchange", Review of Economics and Statistics, 74, 305-14.

[17] Cremer, H. and P. Pestieau (2006), "Wealth transfer taxation: a survey of the theoretical literature", In: Kolm, S.C. and J. Mercier-Ythier (Eds.), Handbook of the Economics of Giving, Altruism and Reciprocity, Volume 2, North-Holland, Chapter 16.

[18] Cremer, H., P. Pestieau and G. Ponthière, (2012), "The economics of long-term care: A survey", Nordic Economic Policy Review, 2, 107-148.

[19] Cremer, H. and Pestieau, P. (2018) "Means-Tested Long-Term Care and Family Transfers", German Economic Review, 19(3), 351-364.

[20] De Nardi, M., E. French and J.B. Jones, (2010), "Why Do the Elderly Save? The Role of Medical Expenses", Journal of Political Economy, 118, 39-75. 
[21] Friend, I. and M. Blume, (1975), "The Demand for Risky Assets", American Economic Review, 65 (5), 900-922.

[22] Guesnerie, R. and J.-J. Laffont, (1978), "Taxing price makers", Journal of Economic Theory, 19, 423-455.

[23] Klimaviciute, J., S. Perelman, P. Pestieau and J. Schoenmaeckers, (2017), "Caring for dependent parents: Altruism, exchange or family norm?", Journal of Population Economics, 30, 835-873.

[24] Koh, S-K. and M. MacDonald, (2006), "Financial Reciprocity and Elder Care: Interdependent Resource Transfers", Journal of Family and Economic Issues, 27, 420-436.

[25] Kotlikoff, L. and A. Spivak, (1981), "The Family as an Incomplete Annuities Market", Journal of Political Economy, 89, 372-91.

[26] Laffont, J-J. and J. Tirole, (1993), A theory of incentives in procurement and regulation, MIT Press, Cambridge MA.

[27] Lewinter, M., (2003) "Reciprocities in care giving relationships in Danish elder care", Journal of Ageing Studies, 17, 357-377.

[28] Light, A. and K. McGarry, (2004), "Why Parents Play Favorites: Explanations for Unequal Bequests ", The American Economic Review, 94, 1669-1681.

[29] Menchik, P.L., (1980), "Primogeniture, Equal Sharing and the U.S. Distribution of Wealth", Quarterly Journal of Economics, 94, 299-316.

[30] Norton, E., (2000), "Long term care", in A. Cuyler \& J. Newhouse (Eds.): Handbook of Health Economics, Volume 1b, chapter 17.

[31] Norton, E., (2016), "Health and long-term care". In: Piggott, J. and A. Woodland (Eds), Handbook of the Economics of Population Aging, Volume 1B, North Holland, Chapter 17. 
[32] Norton E., L.H. Nicholas and S.S-H. Huang, (2013), "Informal Care and Inter-vivos Transfers: Results from the National Longitudinal Survey of Mature Women ", The B.E. Journal of Economic Analysis \& Policy, 14, 377-400.

[33] Norton, E. and D. H. Taylor, Jr, (2005), "Equal Division of Estates and the Bequest Motive", Journal of Aging and Social Policy, 17, 63-82.

[34] Norton, E. and C. H. Van Houtven, (2006), "Inter-vivos Transfers and Exchange", Southern Economic Journal 73, 157-72.

[35] Tirole, J., (1986), "Hierarchies and Bureaucracies: On the Role of Collusion in Organizations", Journal of Law, Economics and Organizations, 2, 181-214.

[36] Tomes, N., (1981), "The Family, Inheritance and the Intergenerational Transmission of Inequality", Journal of Political Economy, 89, 928-58.

[37] Tomini, F., W. Groot and S.M. Tomini, (2016), "Informal care and gifts to and from older people in Europe: The interlinks between giving and receiving", BMC Health Services Research, 16:603. 


\section{Appendix}

\section{A.1 Reformulation of Problem (6)-(8)}

Incentive compatibility implies that, for each type $\beta$ and $\beta^{\prime}$,

$$
\tau(\beta)-v(a(\beta), \beta) \geq \tau\left(\beta^{\prime}\right)-v\left(a\left(\beta^{\prime}\right), \beta\right)
$$

and

$$
\tau\left(\beta^{\prime}\right)-v\left(a\left(\beta^{\prime}\right), \beta^{\prime}\right) \geq \tau(\beta)-v\left(a(\beta), \beta^{\prime}\right) .
$$

Combining these two conditions yields

$$
v\left(a(\beta), \beta^{\prime}\right)-v(a(\beta), \beta) \geq v\left(a\left(\beta^{\prime}\right), \beta^{\prime}\right)-v\left(a\left(\beta^{\prime}\right), \beta\right),
$$

or equivalently

$$
\int_{a\left(\beta^{\prime}\right)}^{a(\beta)} \int_{\beta}^{\beta^{\prime}} v_{a \beta}(x, y) d y d x \geq 0 .
$$

Since $v_{a \beta}<0$, this condition implies that, if $\beta^{\prime} \geq \beta$, $a\left(\beta^{\prime}\right) \leq a(\beta)$. Then, incentive compatibility implies that $a^{\prime}(\beta) \geq 0$.

The first-order condition for truth telling can be written

$$
\tau^{\prime}(\widetilde{\beta})-\left.v_{a}(a(\widetilde{\beta}), \beta) a^{\prime}(\widetilde{\beta})\right|_{\widetilde{\beta}=\beta}=0 .
$$

This equation implies that

$$
\dot{U}_{k}(\beta)=v_{\beta}(a(\beta), \beta) .
$$

This first-order condition, together with $a^{\prime}(\beta)$ are necessary and sufficient conditions for incentive compatibility (Laffont and Tirole, 1993).

Since $\dot{U}_{k}>0$, the only binding participation constraint is the one of the $\underline{\beta}$ type, $U_{k}(\underline{\beta})=0$. This condition, combined with $\dot{U}_{k}(\beta)=v_{\beta}(a(\beta), \beta)$ yields $U_{k}(\beta)=-\int_{\underline{\beta}}^{\beta} v_{\beta} d x$. Consequently, the problem of the parents can be rewritten as (10). 


\section{A.2 Derivation of Equation (11)}

The Hamiltonian function associated with problem (10) is

$$
\begin{aligned}
\mathcal{H} & =w \bar{T}-\pi g-s+(1-\pi) U(s) \\
& +\pi H\left(s+a(\beta)-U_{k}(\beta)-v(a(\beta), \beta)+g\right) f(\beta)-\mu(\beta) v_{\beta}(a(\beta), \beta),
\end{aligned}
$$

where $\mu(\beta)$ is the co-state variable. The first-order conditions are given by

$$
\begin{aligned}
\frac{\partial \mathcal{H}}{\partial a} & =\pi H^{\prime} f(\beta)\left[1-v_{a}(a(\beta), \beta)\right]-\mu(\beta) v_{a \beta}(a(\beta), \beta)=0, \\
\frac{\partial \mathcal{H}}{\partial s} & =-1+(1-\pi) U^{\prime}(s)+\pi \int_{\underline{\beta}}^{\bar{\beta}} H^{\prime} f(\beta) d \beta=0 \\
\frac{\partial \mathcal{H}}{\partial U_{k}} & =\pi H^{\prime} f(\beta)=-\dot{\mu}(\beta) .
\end{aligned}
$$

Using the transversality condition $\mu(\bar{\beta})=0$, equation (A.3) implies

$$
\mu(\beta)=-\int_{\beta}^{\bar{\beta}} \pi H^{\prime} f(s) d s<0 \quad \forall \beta<\bar{\beta}
$$

Using (A.4), (A.1) can be rewritten as

$$
1-v_{a}(a(\beta,) \beta)=\frac{-\int_{\beta}^{\bar{\beta}} H^{\prime} f(s) d s}{H^{\prime} f(\beta)} v_{a \beta}(a(\beta,) \beta)>0 \quad \forall \beta<\bar{\beta} .
$$

\section{A.3 Proof of Lemma 1}

Using (11) and applying the implicit function theorem, we obtain that the sign of $\partial a / \partial g$ is equal to the sign of

$$
\frac{H^{\prime} \int_{\beta}^{\bar{\beta}} H^{\prime \prime} f(s) d s-H^{\prime \prime} \int_{\beta}^{\bar{\beta}} H^{\prime} f(s) d s}{\left[H^{\prime} f(\beta)\right]^{2}}
$$

Then,

$$
\frac{\partial a}{\partial g} \geq 0 \Longleftrightarrow-\frac{H^{\prime \prime}}{H^{\prime}} \leq-\frac{\int_{\beta}^{\bar{\beta}} H^{\prime \prime} f(s) d s}{\int_{\beta}^{\bar{\beta}} H^{\prime} f(s) d s} .
$$

The LHS of the inequality above represents the absolute degree of risk aversion. It is possible to show that

$$
\lim _{\beta \rightarrow \bar{\beta}}-\frac{\int_{\beta}^{\bar{\beta}} H^{\prime \prime} f(s) d s}{\int_{\beta}^{\bar{\beta}} H^{\prime} f(s) d s}=-\frac{H^{\prime \prime}(\bar{\beta})}{H^{\prime}(\bar{\beta})},
$$


so that (A.5) holds with equality at $\beta=\bar{\beta}$. Furthermore, straightforward derivation yields

$$
\frac{\partial-\frac{\int_{\beta}^{\bar{\beta}} H^{\prime \prime} f(s) d s}{\int_{\beta}^{\bar{\beta}} H^{\prime} f(s) d s}}{\partial \beta} \geq 0 \Longleftrightarrow-\frac{H^{\prime \prime}}{H^{\prime}} \leq-\frac{\int_{\beta}^{\bar{\beta}} H^{\prime \prime} f(s) d s}{\int_{\beta}^{\bar{\beta}} H^{\prime} f(s) d s} .
$$

Consider the case where $H($.$) displays DARA. Then, -H^{\prime \prime} / H^{\prime}$ decreases as $\beta$ increases, since dependent parents' consumption increases in $\beta$. Suppose that in this case

$$
-\frac{H^{\prime \prime}}{H^{\prime}} \leq-\frac{\int_{\beta}^{\bar{\beta}} H^{\prime \prime} f(s) d s}{\int_{\beta}^{\bar{\beta}} H^{\prime} f(s) d s} .
$$

Then, (A.7) implies that $-\int_{\beta}^{\bar{\beta}} H^{\prime \prime} f(s) d s / \int_{\beta}^{\bar{\beta}} H^{\prime} f(s) d s$ increases in $\beta$, which is in contradiction with (A.6). Then, under DARA,

$$
-\frac{H^{\prime \prime}}{H^{\prime}}>-\frac{\int_{\beta}^{\bar{\beta}} H^{\prime \prime} f(s) d s}{\int_{\beta}^{\bar{\beta}} H^{\prime} f(s) d s},
$$

implying that $\partial a / \partial g<0$.

\section{A.4 Variation of the uniform policy $g$ with respect to $\alpha$}

Recall that $a(\beta)$ and $s$ are determined by the parents. Consequently they are not directly affected by $\alpha$, but depend indirectly on the weight via its impact on $g$. With this in mind, totally differentiating (13) yields

$$
\frac{\partial g}{\partial \alpha}=\frac{\pi\left[1-\int_{\underline{\beta}}^{\bar{\beta}} H^{\prime} f(\beta) d \beta\right]+\int_{\underline{\beta}}^{\bar{\beta}}(1-F(\beta)) v_{a \beta} \frac{\partial a(\beta)}{\partial g} d \beta}{-S O C_{g}},
$$

where $S O C_{g}$ denotes the second derivative of the government's problem with respect to

$g$. The expression above is always negative under DARA, since $\int_{\beta}^{\bar{\beta}} H^{\prime} f(\beta) d \beta>1$ and $\partial a(\beta) / \partial g<0$ for all $\beta$.

\section{A.5 Derivation of Equation (15)}

The Hamiltonian corresponding to Problem (14) is 


$$
\begin{aligned}
\mathcal{H} & =(1-\alpha)[w \bar{T}-\pi g(\beta) f(\beta)-s+(1-\pi) U(s)] \\
& +\pi(1-\alpha)\left[H\left(s+a-v(a, \beta)-U_{k}(\beta)+g(\beta)\right) f(\beta)\right] \\
& +\alpha \pi U_{k}(\beta) f(\beta)-\mu_{\beta}\left[-v_{\beta}\right] .
\end{aligned}
$$

The first-order conditions are given by

$$
\begin{aligned}
& \frac{\partial \mathcal{H}}{\partial a}=\pi(1-\alpha) H^{\prime} f(\beta)\left[1-v_{a}(a(\beta), \beta)\right]-\mu(\beta) v_{a \beta}(a(\beta), \beta)=0, \\
& \frac{\partial \mathcal{H}}{\partial g}=-(1-\alpha)\left[\pi f(\beta)+\pi H^{\prime} f(\beta)\right]=0, \\
& \frac{\partial \mathcal{H}}{\partial U_{k}}=\pi(1-\alpha) H^{\prime} f(\beta)-\alpha \pi f(\beta)=-\dot{\mu}(\beta) .
\end{aligned}
$$

Condition (A.10) reduces to $H^{\prime}=1$ for all $\beta$. This implies that the consumption of dependent parents $m(\beta)=H^{-1}(1)$ which does not depend on the children's type. This means that the risk of dependence is fully insured. Using this condition and the transversality condition $\mu(\bar{\beta})=0$, equation (A.11) implies

$$
\mu(\beta)=-\pi(1-2 \alpha)[1-F(\beta)]
$$

Then, (A.9) can be rewritten as

$$
1-v_{a}(a(\beta), \beta)=-\frac{1-F(\beta)}{f(\beta)} \frac{(1-2 \alpha)}{(1-\alpha)} v_{a \beta} .
$$

